\title{
Detection of railway subgrade moisture content by GPR
}

\author{
Sixin Liu *, Jiaqi Cai, Lei Fu \\ College of Geo-exploration Science and Technology, Jilin \\ University, 130061, Changchun, China \\ 543144037@qq.com, liusixin@jlu.edu.cn
}

\author{
Yanqian Feng \\ The 3rd Railway Survey and Design Institute Group \\ Corporation, 300000, Tianjing, China
}

\begin{abstract}
In cold regions, heaving of the railway subgrade is an important security risk to high-speed railway. It is necessary to find out the moisture content of the subgrade to secure the normal railway operation. Common Mid-Point (CMP) measurement using Ground Penetrating Radar (GPR) was carried out to estimate the moisture content of railway subgrade. As the subgrade is layered medium typically, CMP method is suitable for moisture content measurement. At first, the CMP data is analyzed through velocity spectrum to obtain the stacking velocity, from which the vertical interval velocity profile can be calculated by Dix's formula. The moisture content can be obtained by Topp's formula from dielectric constant which depends on the velocity. However, the velocity analysis has lots of problem for the shallow and thin layers like rail subgrade. With the help of FDTD simulation by gprMAX, we find that there are many multiples and refracted waves which cause much false results during velocity analysis as the offset is large. Under the guidance of the simulated result, we use optimized data gathers for velocity analysis to the real data. It is found that the final result is good and in accordance with the result of induced polarizability (IP). It is proven that it is indeed feasible to use GPR to detect moisture content of subgrade. This method can be widely applied in subgrade moisture detection.
\end{abstract}

Keywords-ground penetrating radar (gpr), velocity spectrum, velocity analysis, common mid-point (cmp), moisture content

\section{INTRODUCTION}

With the social development in China, the high-speed railway has become a part of our life. However, the moisture content of railway subgrade can't be neglected during the construction and operation of high-speed railway, especially in cold regions. As we know, the volume becomes large as water becomes ice, and that is why frost heaving always takes place in cold winter and may cause a huge safety problem to the train in high speed. It's necessary to know the underground moisture content, to avoid the blindness of construction and make sure of safety operation. Drilling is the only option to get accurately water content at present ${ }^{[1]}$, which is intrusive, costly and time consuming.. Although the IP is reliable method for geophysical exploration, it only calculates the value of polarizability which is somehow related to water content. GPR is a non-destructive method to detect moisture content; the fundamentals are Topp's and Dix's formula ${ }^{[2,3]}$.

Ground penetrating radar (GPR), as a geophysical tool for the nondestructive detection of shallow subsurface layers by electromagnetic waves, is widely used in the field of environmental and engineering problems. Using groundpenetrating radar to measure the moisture content has become an important branch of GPR applications ${ }^{[4]}$. Grit and Ygur realized the measurement of moisture content of porosity by combining radar and geoelectric method ${ }^{[5]}$. In recent years, a lot of papers using GPR to measure soil moisture content have been published ${ }^{[6-13]}$.

It is essential to estimate the electromagnetic velocity, in order to access the water content. The velocity analysis is an accurate way, which originated from seismic exploration ${ }^{[14]}$. However, there exist special problems in the process of applications. Feng improved signal to noise ratio by CMP antenna array and data processing technology, and got a good result in velocity analysis, which is applied to the landmine detection successfully ${ }^{[15]}$. Liu obtained the electromagnetic wave velocity through the envelope velocity analysis method, which can monitor the underground dynamic water level ${ }^{[16]}$. Recently many papers about electromagnetic wave velocity estimation are published ${ }^{[17-21]}$. Lu discussed the soil moisture content estimation and monitored the groundwater level $\mathrm{el}^{[22-23]}$. Liu used GPR profiles and moisture content to estimated hydraulic conductivity parameters, and accurately distinguished fluctuations of of groundwater level ${ }^{[24]}$. But there is no results dealing with very shallow layered structure in these papers. In shallow formations, routine velocity analysis is no longer suitable.

In this paper, the problem of shallow and thin layer has been analyzed and solved under the guidance of numerical simulation by GPRMAX ${ }^{[25]}$. In addition, a series of CMP data are used to obtain the moisture content at a point, which can be interpolated, and get a long moisture content profiles.

\section{CONVENTIONAL THEORY}

\section{A. Theory of Velocity Analysis and Velocity Spectrum}

For horizontal layered medium like railway subgrade, the function between reflected wave time $\Delta t_{i}$, and shot-geophone distance $\mathrm{x}_{i}$, root-mean-square velocity $\mathrm{v}_{\sigma}$ are given for each time $t_{0}$ by

$$
\Delta t_{i}=\Delta t_{i}\left(t_{0}, x_{i}, v_{\sigma}\right)=\sqrt{t_{0}^{2}+\frac{x_{i}^{2}}{v_{\sigma}^{2}}}-t_{0}
$$


We can calculate of reflection wave time-distance curve at a certain velocity, by fixing the value of echo time $t_{0}$. According to the time-distance curve equation, the values are obtained from CMP data and then accumulate together to calculate the stacking amplitude. If the corresponding curve at a certain velocity coincides with the event of reflection wave at the $t_{0}$ time, then the value of stacking amplitude must be the largest. Different velocity values were tested and after calculation of maximum stacking amplitude and its corresponding velocity, we can find the velocity at $t_{0}$. This is the principle of stacking velocity spectrum. We can obtain the interval velocity from the stacking velocity by Dix's formula.

\section{B. Topp's Formula}

According to the actual situation of the subgrade, the thickness of subgrade layer is $\sim 2.5 \mathrm{~m}$, which consists of ballast, gravel and others. We can obtain moisture content by using the Topp's formula

$$
\theta=-5.3 \times 10^{-2}+2.292 \times 10^{-2} \varepsilon-5.5 \times 10^{-4} \varepsilon^{2}+4.3 \times 10^{-6} \varepsilon^{3}
$$

\section{PROBLEMS OF MULTIPLE WAVES AND REFRACTEDWAVES IN LAYERED STRUCTURES}

\section{A. Railway Subgrade}

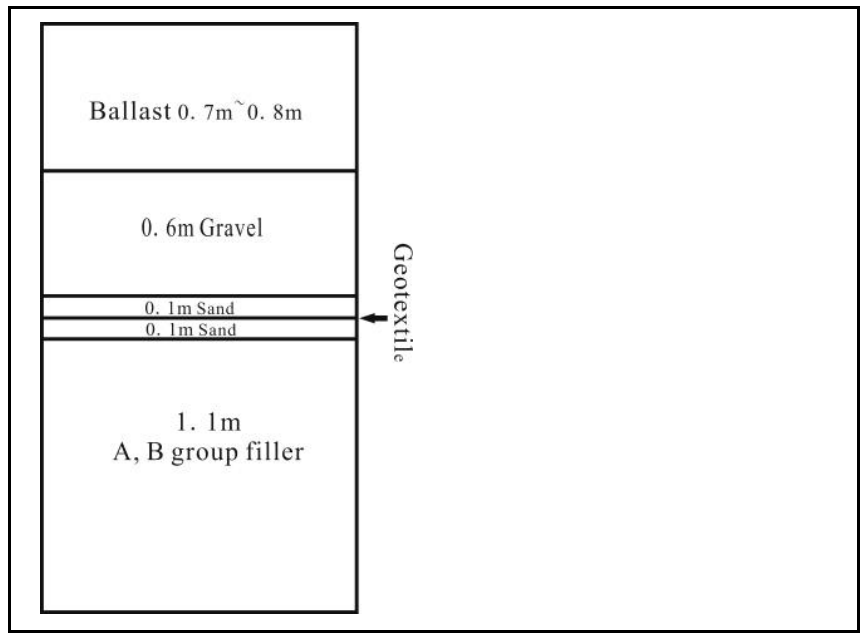

Fig.1 Map of railway subgrade

Fig. 1 shows a typical railway subgrade structure, which can be roughly divided into 4 layers, i.e., ballast, gravel, sand and A, B group filler from top to bottom. We found there are artifacts in the velocity analysis formerly, when the railway subgrade layer is shallow and thin. Therefore, we try to build a numerical subgrade model and analyze the measured data.

\section{B. The Multiple Waves and Refracted Waves}

The numerical model is shown in Fig. 2 which is corresponded to the real model in Fig. 1. The blue part is a thin layer with thickness of $0.7 \mathrm{~m}$ and the dielectric constant of 4. The light blue layer is $0.6 \mathrm{~m}$ thick and the dielectric constant is 6 . The thickness of the orange layer is $1.3 \mathrm{~m}$ and its dielectric constant is 8.5. The white part represents air layer and the red part stands for stratum with the dielectric constant of 10. We use gprMAX for numerical simulation. The grid size is $0.05 \mathrm{~m}$, the emission source is Ricker wavelet with peak frequency of $100 \mathrm{MHz}$, the recording time window is $120 \mathrm{~ns}$, the offset increament is $0.2 \mathrm{~m}$. we take the point $(15,12)$ as the measuring point and collect 64 traces with the CMP method.

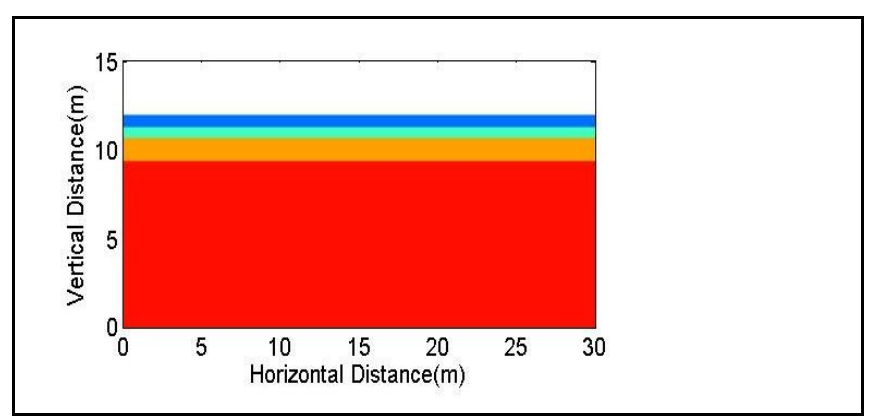

Fig.2 Relative dielectric constant model

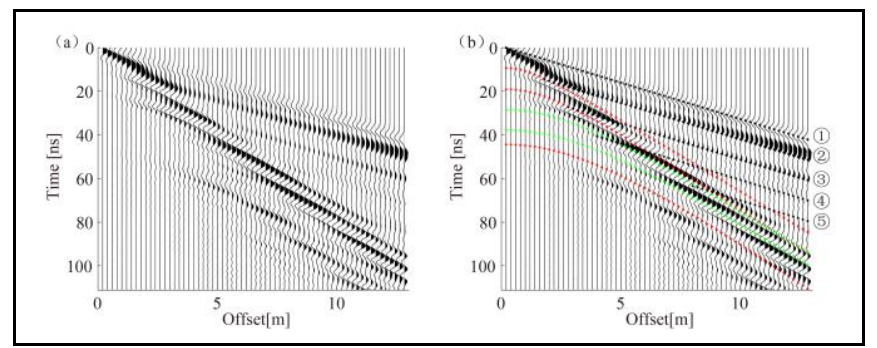

Fig.3 (a) The common midpoint radar profile. (b) The position of direct waves, reflected waves and multiple waves.

Fig. 3a is the simulated result, which shows a number of discontinuous events. In order to facilitate the analysis, curves with different color are plotted in Fig. $3 b$ to mark these events. The black line at the upper right corner in Fig. 3b represents the air wave. And the next four curves are refracted waves, which reflected from underground at different times and their amplitudes are decreased in turn. The first green line represents multiple waves, which crosses the first layer twice and the second layer once. And its energy is mainly distributed in the range of 5-12.8m. The second green one crosses the first layer three times and the second layer once, and its energy is mainly distributed in the range of $9-12.8 \mathrm{~m}$. The three red lines are the reflection waves from different layers, which we care about. The first red line represents the first interface, which is mainly distributed in the range of $1.2-5 \mathrm{~m}$. It seems that different wave dominates in different range. Multiple and refracted waves obscure the reflected wave we are interested in.

The reasons for this phenomenon are as follows. Frist, when the angle of the reflection becomes larger, the energy of multiple waves becomes lager, and the reflection energy becomes weaker until it can't be detected. Second, if the reflection angle is small, the energy of the direct wave is so strong that the energy of the reflected wave is weak. The reflected event of the second layer appears in the range of 2$11 \mathrm{~m}$, although it forms in the deeper place, but similar phenomenon also exists. Compared with the first and second layers, the energy of the third reflection wave is weaker, but 
its event is complete after proper gain. In summary, we can draw a conclusion that multiple and refracted waves mainly exist in the shallow layers.

Fig.4a, b and c are obtained by analyzing 1-64 traces, 3-23 traces and10-35 traces, respectively. However, we can't get what we want from Fig.4a. In contrast, we can get information of first layer from Fig. 4b, second layer and third layer from Fig.4c. In Fig. 5, the blue line is the theoretical value, and the green line represents the velocities which get from the velocity analysis. It is easy to find out that the velocities got by velocity analysis are basically in accordance with the theoretical velocities. However, there is a little difference in time. The reason of this is that velocity analysis generally takes the peak value of the signal, but the theoretical value is the jumping point of signal.

Therefore, in velocity analysis, selection of appropriate traces to analyze velocity spectrum to get meaningful information about the railway subgrade structure. What's more, we can also get interval velocity through combing results of several times of velocity analysis with different trace range.

\section{APPLICATIONS}

The field data were measured in certain railway in China, and SIR-20 system and 100MHZ antenna were used for field survey. We used common middle point method to get the related information: the offset distance is $0.2 \mathrm{~m}$, the time window is $120 \mathrm{~ns}$ and the number of sampling points is 512 .

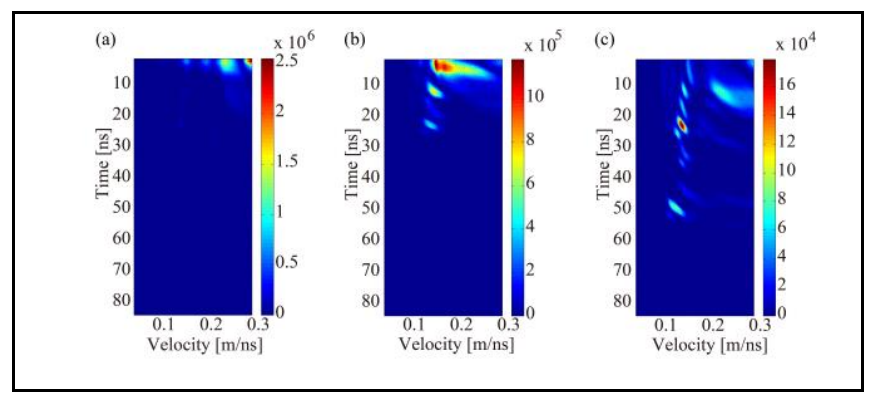

Fig.4 Velocity spectrum. (a) All CMP traces. (b) 2-23 traces. (c) 10-35 traces.

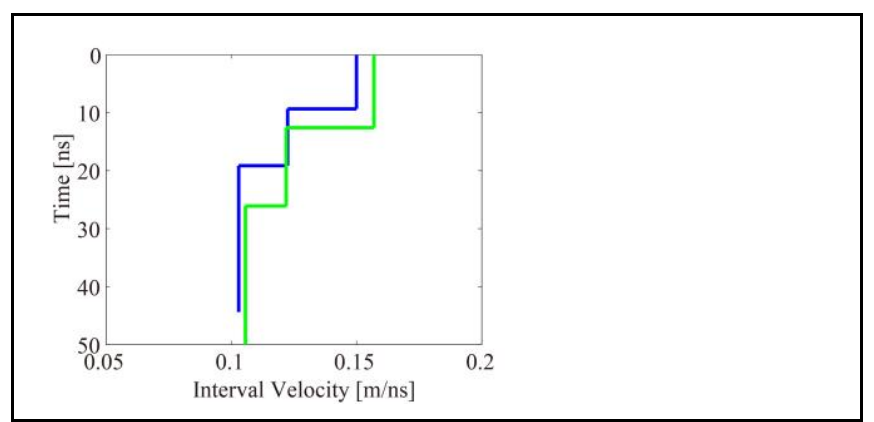

Fig.5 Vertical distribution of velocity

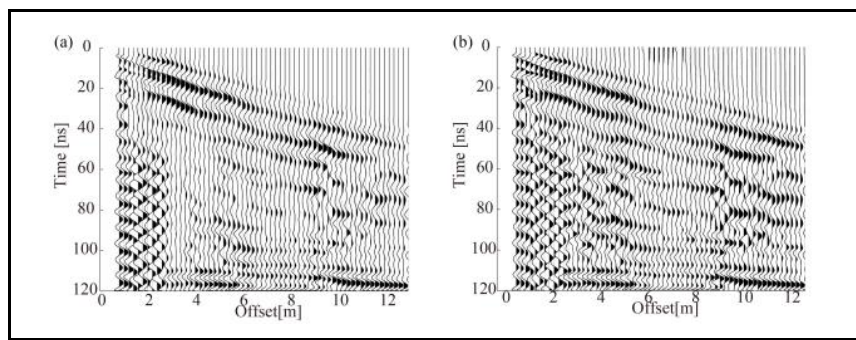

Fig.6 (a) Original common offset radar profile. (b) After preprocessing.

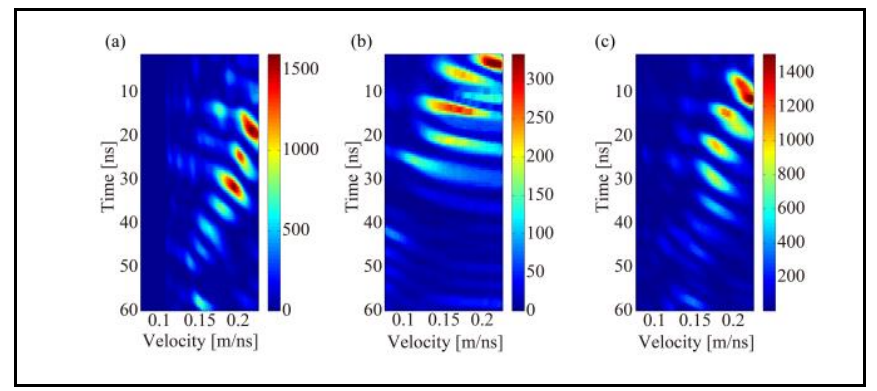

Fig.7 Velocity spectrum. (a) All CMP traces. (b) 1-13 traces. (c) 1-35 traces.

Fig. 6a is the original CMP profile and processing is necessary because of its low signal-to-noise ratio. The data processing step includes: zero time correct, direct filter, gain control, and frequency domain filtering. The process CMP profile is shown in Fig. 6 b.

We analyze the data by using different traces under the guidance of numerical simulation and the results are showed in Fig.7. The information of velocity spectrum is obtained according to the condition of subgrade and the position of reflecting layer by choosing different traces. Fig. 7a shows the result of velocity analysis by using 1-64 traces, from which we can't get the corresponding energy at the nearby of the layer boundary. In order to verify this, the time-distance curves are given for velocity information obtained in the Fig.7a by the red lines in the Fig.8a. We can find that the refracted wave is the source of the errors. The error energy response information is shown in TABLE I .

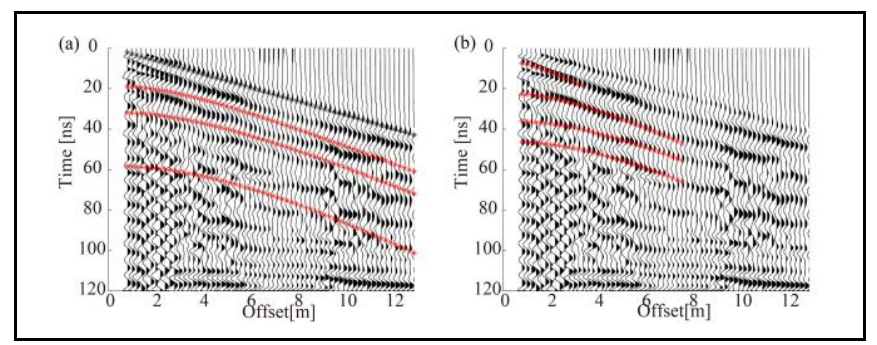

Fig.8 Profiles of common midpoint radar. (a)Red lines represent error velocities. (b)Red lines represent right velocities 
TABLE I.

INFORMATION OF ERROR ENERGY

\begin{tabular}{|l|l|l|}
\hline \multicolumn{1}{|c|}{ Number of layer } & \multicolumn{1}{|c|}{ time(ns) } & \multicolumn{1}{|c|}{ Velocity(m/ns) } \\
\hline 1 & 18.6 & 0.22 \\
\hline 2 & 31.7 & 0.198 \\
\hline 3 & 58.3 & 0.154 \\
\hline
\end{tabular}

We pick up the information of the first layer from traces no 1-13. Nevertheless, the second layer and the third layer are influenced by many factors, thus we obtain the second and third layer velocity information from traces 1-35. The information is expressed by red lines in Fig. 8b. We can find that red line is coincident with events of small offset.

As there are too many CMP points along the railway line, Fig. 9 shows the velocity spectrum of the first 6 measurement points. The velocity spectrum obtained in this way can reflect the underground structure well. We pick up the stacking velocities at 9 12ns, 20 25ns and 40 50ns respectively according to the actual situation of the subgrade. Then those can be converted to interval velocities by Dix's formula. Thus we can obtain the dielectric constant from the interval velocity, and we can obtain the underground moisture content by using Topp's formula. After getting a series of moisture content of the underground, we can draw an underground moisture content profile by using interpolation as show in Fig. 10a.

TABLE II

INFORMATION OF RIGHT ENERGY

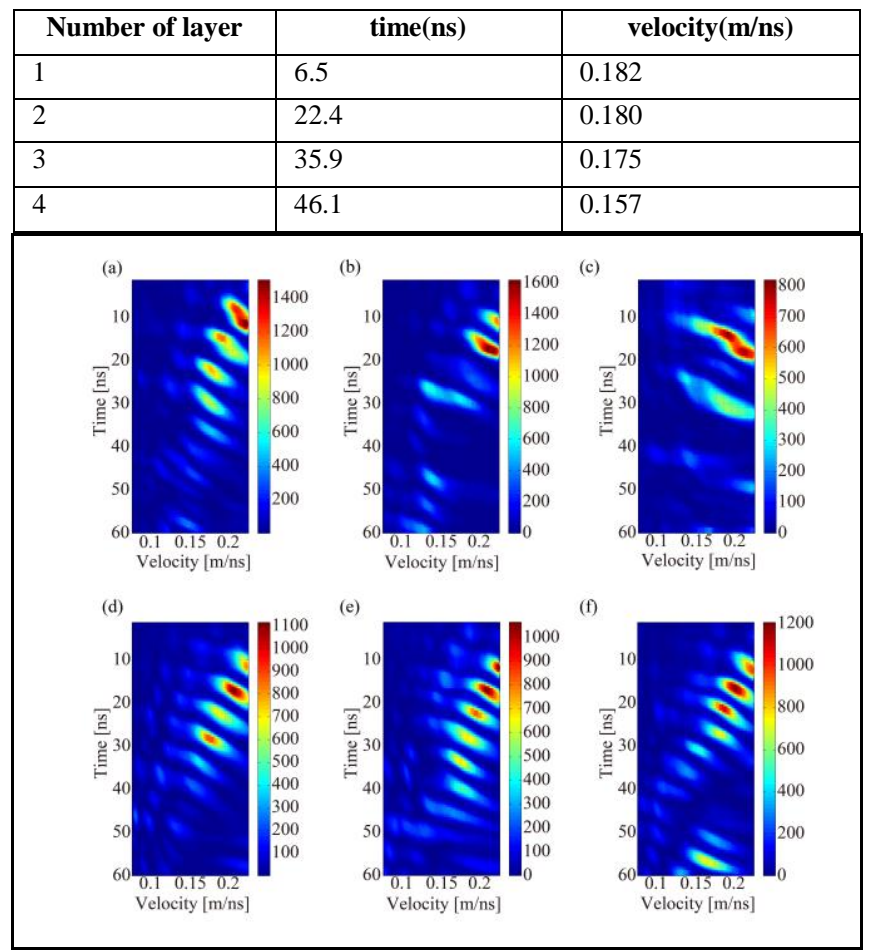

Fig.9 Velocity spectrum of the first six measuring points. (a) The first point. (b) The second point. (c) The third point. (d) The fourth point. (f) The fifth point.

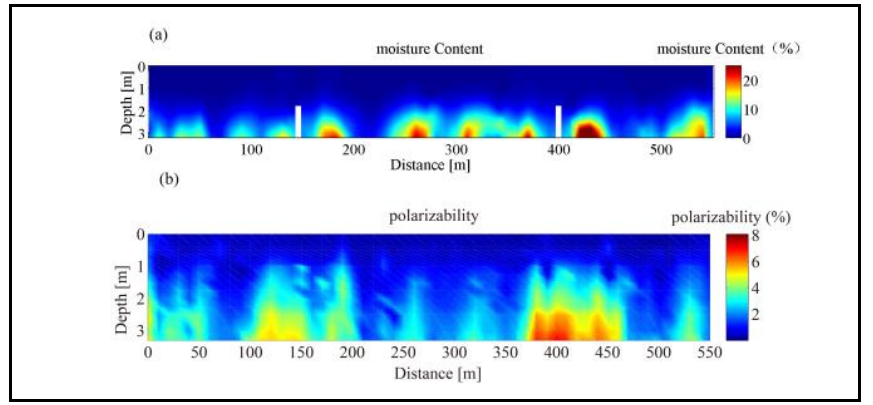

Fig.10 (a) Water content profile. The blank blocks are locations of culverts. (b) The map of IP

In Fig.10a, there are many numbers of the anomaly positions, which are mainly located at 10-50, 90, 130 - 140, $180-190,260-270,310,370,420-450,520-540 \mathrm{~m}$. We have known that there are two $4 \mathrm{~m} * 4 \mathrm{~m}$ culverts at $150-160$ and 400 410 meters underground, respectively. The locations of culverts are represented by blank blocks in Fig.10a. As it is known to all, the internal components of the culverts are air. However, the effect of air on radar is high speed and low dielectric constant. Definitely, it must affect the values of moisture content at the positions of 150-160 and 400-410 meters, even the surroundings.

According to the IP result shown in Fig. 10b, locations at $0-50,100-190,260,310,370-450$ and $540 \mathrm{~m}$ are mainly indicating the anomalous positions. As we know, there is a correlation between the IP result and moisture content, so it is similar with the anomaly positions in Fig. 10a. In addition, there is difference between the moisture content profile and the polarization profile at the position of 90 meters. The rest of the anomaly positions are consistent for the results from two methods. Through the comparison of IP, we find that the method of using GPR to measure the moisture content of subgrade is feasible. At the same time, it further proves that velocity analysis by selecting the optimize CMP traces is necessary.

\section{CONCLUSION}

The multiple and refracted waves appeared in the CMP data have great influence on CMP velocity analysis based the reflection waves in shallow layer. According to this phenomenon, the optimal traces ranges are used for velocity analysis, which is applied in the actual data processing. The moisture content result obtained through the proposed method is reliable through comparing with IP data. It is proven that GPR is feasible to measure the moisture content of subgrade. Thus, it will be widely used during railway construction and operation in the cold regions.

\section{ACKNOWLEDGMENT}

This work is supported by "College of Geo-exploration Science and Technology, Jilin University" and "The 3rd Railway Survey and Design Institute Group Corporation". 


\section{REFERENCES}

[1] Andrew C. Brown, Gregory Dellinger, Ali Helwa, Chadi El-Mohtar, Jorge Zornberg, Robert B. Gilbert, "Monitoring a Drilled Shaft Retaining Wall in Expansive Clay: Long-Term Performance in Response to Moisture Fluctuations," American Society of Civil Engineers, 2015.

[2] Topp, G Davis, Annan A, "Electromagnetic determination of soil water content: Measurements in coaxial transmission lines: Water Resour Res,"Water Resources Research, vol.16, pp. 574-582, 1980.

[3] Dix C H, "Seismic velocities from surface measurements," Geophysics, vol. 20, pp. 68-86, 1955.

[4] Harry M. Jol, Ground Penetrating Radar Theory and Applications, Eau Claire, 2009, pp. 1-56.

[5] Grit D, Ugur Y, "Estimation of water of water content and porosity using combined radar and geoelectrical measurements," European journal of environmental and engineering geophysics, vol. 1, pp. 71-85, 1999.

[6] Annan A P, Cosway S W, Redman J D, "Water table detection with ground penetrating radar," Seg Extended Abstracts, vol. 10, pp. 494-496, 1991.

[7] Tsoflias G P, Halihan T, Sharp J M, "Monitoring pumping test response in a fractured aquifer using ground penetrating radar," Water Resources Research, vol.37, pp. 1221-1229, 2001.

[8] Pyke K, Eyuboglu S, Daniels J J , M Vendl, K Pyke, A controlled experiment to determine the water table response using ground penetrating radar," Journal of Environmental and Engineering Geophysics, vol.13, pp. 335-342, 2012.

[9] Idi B Y, Kamarudin N, "Ground water estimation and water table detection with ground penetrating radar," Asian Journal of Earth Science, vol.4, pp. 193-202, 2011.

[10] Seger M A, "Detection of water-table by using ground penetration radar (GPR)," Eng. and Tech. Journal, vol.29, pp.554-566, 2011.

[11] Yuichi N, "Estimation of groundwater level by GPR in an area with multiple ambiguous reflections," Journal of Applied Geophysics, vol.47, pp. 241-249, 2001.

[12] J A Huisman, S S Hubbard, J D Redman, "Measuring soil water content with ground penetrating radar," Vadose Zone Journal, vol. 2,pp.476-491, 2013
[13] Ma Fujian, SG Lei, S Yang, F Zhen, Y Wang, "Study on the relationship between soil water content and ground penetrating radar signal properties," Chinese Journal of Soil Science, vol.4, pp. 809-815, 2014.

[14] D L Macedo, JJSD Figueierdo, RF Portugal, "Velocity analysis on CMP sections based on the smearing paradigm," SEG Technical Program Expanded Abstracts, vol.28, pp. 228-341, 2009.

[15] Feng X, Sato M, Zhang, "CMP antenna array GPR and signal-to clutter ratio improvement," IEEE Geoscience and Remote Sensing Letters, vol.6, pp. 23-27, 2009.

[16] Liu H, Xie X, Cui J, “Groundwater Level Monitoring for Hydraulic Characterization of an Unconfined Aquifer by Common Mid-point Measurements using GPR," Journal of Environ mental and Engineering Geophysics, vol.19, pp. 259-268,2014.

[17] Jacob R W, Hermance J F, “Assessing the precision of GPR velocity and vertical two-way travel time estimates," Journal of Environmental and Engineering Geophysics, vol.9, pp. 143-153, 2004.

[18] Lu Q, Quantitative analysis for hydrogeology and soil contamination by ground penetrating radar [Ph.D. thesis], Sendai, Japan: Tohoku University, 2005.

[19] Liu H, and Sato M, In situ measurement of pavement thickness and dielectric permittivity by GPR using an antenna array. NDT \& E International, vol.64, pp. 65-71, 2014.

[20] Liu H, Takahashi K, and Sato M, "Measurement of dielectric permittivity and thickness of snow and ice on a brackish lagoon using GPR," IEEE Journal of Selected Topics in Applied Earth Observations and Remote Sensing, vol.7, pp. 820-827,2014.

[21] Liu H, Sato M, "Dynamic Groundwater Level Estimation by the Velocity Spectrum Analysis of GPR," International Conference on Ground Penetrating Radar, pp.413-418, 2012.

[22] Lu Q, "Monitoring of subsurface environment by ground penetrating radar," [M.S. thesis], Sendai, Japan: Tohoku University, 2002.

[23] Lu Q, M Sato, "Groundwater monitoring by GPR in mongolla", 9th International Conference on Ground Penetrating Radar, pp. 545-550, 2002 .

[24] Liu H, Sato M, "Groundwater Level Monitoring for Hydraulic Characterization of an Unconfined Aquifer by Common Mid-point Measurements using GPR," Journal of Environmental and Engineering Geophysics. Vol.19, pp. 259-268, 2014.

[25] Warren, Giannopoulos, Giannakis, "An advanced GPR modelling framework: The next generation of gprMax," Advanced Ground Penetrating Radar (IWAGPR), 2015 8th International Workshop on, IEEE,2015 